\title{
EL APRENDIZAJE SIGNIFICATIVO EN LA ENSEÑANZA DEL DIBUJO.
}

\author{
SIGNIFICANT LEARNING IN TEACHING DRAWING
}

FECHA DE RECIBO: OCTUBRE 31, 2012

FECHA DE ACEPTACIÓN: DICIEMBRE 20, 2012

\section{FREDY LOAIZA DE LA HOZ}

Arquitecto - Especialista en estudios pedagógicos. Profesor Catedrático del Área de Expresión. Programa de Diseño de Modas y Alta Costura, Universidad Autónoma del Caribe. frelode@gmail.com

\section{RESUMEN:}

Ante la problemática que presenta un gran número de estudiantes que ingresan a los primeros semestres de los distintos programas de la facultad en los cursos del área de expresión, se hace necesario por parte de los docentes, la aplicación de estrategias pedagógicas que posibiliten a sus educandos, la aplicación y desarrollo de aprendizajes significativos. Elló, consiste en relacionar el conocimiento previo con la nueva enseñanza, es decir, referenciar lo que ya sabe con lo que se le está enseñando. Con el presente artículo, se pretende contribuir a la sensibilización de todos los involucrados en este proceso con el fin de tratar de elevar los niveles académicos de los estudiantes y entregar a la sociedad un profesional que cumpla con el perfil estipulado institucionalmente.

\section{PALABRAS CLAVE:}

Aprendizaje significativo, Abstracción, Desarrollo cognitivo, Paradigma constructivista, Memorización comprensiva, Relaciones sustantivas, Significatividad Lógica

\begin{abstract}
:
Beginning career students in faculty of Architecture, Art and Design have a lot of problems with their performance in drawing and graphic expression. Teachers should implement instructional strategies in order to aim significant learn linking prior knowledge with new teaching. This article aims to sensitize everyone involved in this process and to explore new ways to rise students educational level.
\end{abstract}

\section{KEYWORDS:}

Significant learn, Abstraction, Cognitive development, Constructivist paradigm, Comprehensive memorization, Substantive relation, Logical significance 
ARTE \& DISEÑO, ISSN: 1692-8555, Vol. 10 Núm. 2, Julio - Diciembre de 2012

FREDY LOAIZA DE LA HOZ

\section{INTRODUCCIÓN}

Un gran número de estudiantes que ingresan a los primeros semestres de los distintos programas de la Facultad de Arquitectura, Arte y Diseño de la Universidad Autónoma del Caribe evidencian significativas carencias de conceptos básicos y serias deficiencias en la fundamentación para graficar y representar sus ideas. Con el fin de contribuir a fortalecer la calidad de los procesos metodológicos y de las practicas pedagógicas de los cursos relacionados con la expresión gráfica, se hace necesario brindar elementos teóricos y prácticos que estimulen el desarrollo de la creatividad del estudiante haciéndolo consciente de la realidad social de su contexto local, regional y nacional para que se involucre como sujeto en la trasformación y desarrollo de la sociedad en que esta inmerso.

Se plantea la actitud de cambio que debe asumir el docente frente a las innovaciones en los procesos educativos, donde el espíritu reflexivo orientado al logro de la autonomía personal en un marco de libertades, de enseñanza, de aprendizaje, de investigación y cátedra, sirva para la integración de la comunidad educativa y para el aprendizaje significativo.

La argumentación técnica que fundamenta las estrategias para el aprendizaje de los cursos en el campo de la expresión gráfi$\mathrm{ca}$, permite la cualificación sobre aprendizaje significativo desde la pedagogía activa, que centra al estudiante como ser integral y protagonista principal de los procesos de aprendizaje y en la construcción de conocimientos. A su vez, compromete a docentes y estudiantes con una cultura investigativa que genere producción de conocimientos válidos para uno y otro, al docente en el enriquecimiento creativo de su quehacer pedagógico y al estudiante en la transformación de su contexto social, promoviendo así la función social de las instituciones de educación superior.

\section{EL APRENDIZAJE SIGNIFICATIVO EN LA ENSEÑANZA DEL DIBUJO.}

Se ha observado con mucha preocupación que a algunos estudiantes que ingresan a los primeros semestres, se les dificulta la comprensión de los contenidos básicos relacionados con el dibujo, la creatividad y la estética para la presentación de los trabajos prácticos, que a la postre son la descripción y el vocabulario gráfico con que un diseñador describe y transmite su creación, por lo cual, se produce una distorsión en el mensaje que se desea llegue a todos sus interlocutores que ahora son los profesores y compañeros, pero más adelante serán sus potenciales clientes.

La mayoría deinstituciones de educación superior, formales y no formales, no practican un examen de aptitudes y habilidades gráficas que permita la selección de estudiantes con conocimientos y alguna práctica en el dibujo. Además, algunos muestran poco interés por aprender los conceptos fundamentales de esta área, porque consideran que no están al alcance de su comprensión, presentando muchas limitaciones en los niveles de interpretación y abstracción, dificultándoseles el proceso del desarrollo cognitivo y la construcción de aprendizajes significativos importantes que se reflejarán en posteriores períodos académicos y por ende, en la calidad de los profesionales que egresarán de los diferentes programas.

\section{" La mayoría de Instituciones de educación superior, formales y no formales, no practican un examen de aptitudes $y$ habilidades gráficas que permitan la selec- ción de estudiantes con conocimientos y alguna práctica en el dibujo."}

Otras consecuencias de esta carencia de conceptos previos son, primero, la imposibilidad de elaboración de trabajos con honestidad, limitándose a copiar y/o calcar trabajos de los compañeros, y segundo, la pérdida de asignaturas o en el peor de los casos, la deserción.

Razones como las anteriores conducen a reflexionar y a evidenciar los compromisos que deben asumir los profesores del área de expresión en los primeros semestres en la orientación de los aprendizajes que posibiliten un conocimiento profundo y perdurable, y desarrollen destrezas y habilidades propias de todo ilustrador; además, resaltar la importancia de la participación activa de los educandos para la construcción de aprendizajes significativos, considerándose éstos como (Borja, 1990)" la acomodación de nuevos conocimientos a partir de los conocimientos previos, dándole significado propio" (p.21). Por lo tanto, es deber del docente investigar, planear y organizar estrategias adecuadas a las necesidades particulares de los estudiantes y del área que se trabaja, lo que es coherente con el paradigma constructivista que da lugar a elaborar teorías sobre el aprendizaje relacionado con la realidad del campo educativo.

Hablar de aprendizaje significativo equivale, según Ausubel (1976), a "en poner de relieve el proceso de construcción de significado como el elemento central del proceso de enseñanza y aprendizaje. El estudiante no aprende un contenido cualquiera, un concepto, una explicación de un fenómeno físico o social, un procedimiento para resolver determinado tipo de problemas, una norma de comportamiento, etcétera, cuando no es capaz de atribuirle un significado. De hecho, en sentido estricto, el estudiante puede aprender también estos contenidos sin atribuirles significado alguno; es lo que sucede cuando aprende de una forma puramente memorística y es capaz de repetirlos o de utilizarlos mecánicamente sin entender en absoluto lo que está diciendo o haciendo". (p.46) La mayoría de las 
ARTE \& DISEÑO, ISSN: 1692-8555, Vol. 10 Núm. 2, Julio - Diciembre de 2012 El aprendizaje significativo en la enseñanza del dibujo, págs. 49 - 52

veces, lo que sucede es que el estudiante es capaz de atribuir únicamente significados parciales a lo que aprende, no significa exactamente lo mismo para el profesor que lo ha enseñado, que para él que lo ha aprendido, no tiene las mismas implicaciones ni el mismo poder explicativo para ambos, que no pueden aplicarlo o utilizarlo en igual extensión y profundidad.

Este cambio de perspectiva es importante porque subraya el carácter abierto y dinámico del aprendizaje, y plantea el problema de la dirección o direcciones en las que debe actuar la enseñanza para que los estudiantes profundicen y amplien los significados que construyen mediante su participación en las actividades de aprendizaje.

¿Qué quiere decir que los estudiantes construyen conocimiento significativo? Ausubel (1976), afirma: "los estudiantes construyen conocimientos significativos cada vez que son capaces de establecer relaciones sustantivas y no arbitrarias entre lo que aprenden y lo que ya conocen" (p.10). Por ejemplo, para dibujar formas complejas, éstas se enmarcan en figuras geométricas sencillas para tener un pleno dominio de las proporciones, luego entonces, el estudiante debe saber diferenciar un rectángulo de un cuadrado, y estar en capacidad de deducir, en un rectángulo, cuál es su proporción, es decir, su relación alto-ancho. Esto quiere decir que el estudiante debe poseer conocimientos previos de geometría elemental. De igual forma debe aplicar la habilidad y la destreza en el desarrollo de los trazos, aptitudes estas adquiridas previamente mediante la realización de ejercicios de fundamentación, elaborados con entusiasmo y a conciencia.

En términos de Piaget, se podría decir que (Daz Barriga, 1998) "construye significados integrando o asimilando el nuevo material de aprendizaje a los esquemas que ya poseen sobre la realidad. Esta construcción de significados implica igualmente una acomodación, una diversificación y una mayor interconexión de los sistemas previos. Al relacionar lo que ya se sabe con lo que se está aprendiendo, los esquemas de acción y de conocimiento se modifican y al modificarse, adquieren nuevas potencialidades como fuente futura de atribución de significados; en muchas ocasiones, el aprendizaje se limita a la mera repetición memorística" (p.72).

El aprendizaje significativo, según Ausubel (1976), “plantea una serie de exigencias; ante todo es necesario que el nuevo material de aprendizaje sea potencialmente significativo, es decir, que sea susceptible de dar lugar a la construcción de aprendizajes significativos. Para ello debe cumplir dos condiciones, una intrínseca al propio contenido de aprendizaje y la otra relativa al estudiante particular que va a aprenderlo" (p.310).

La primera condición hace referencia a que el contenido posea una cierta estructura interna, una cierta lógica intrínseca, un significado en sí mismo. El estudiante difícilmente podrá construir aprendizajes significativos si el contenido de aprendizaje de es vago o está poco estructurado, es decir, si no es potencialmente significativo desde el punto de vista lógico., al decir de Díaz Barriga (1998), “esta significatividad Lógica, como la denomina Ausubel, no sólo depende de la estructura interna del contenido, sino también de la manera como éste se le presenta al estudiante" (p.73).

\section{"La activación del conocimiento previo puede servir al profesor en un doble sentido: para reconocer lo que saben sus alumnos y para utilizar tal conocimiento como base para promover nuevos aprendizajes."}

La segunda condición para que el material sea potencialmente significativo es que el estudiante relacione de forma no arbitraria la nueva información con la que ya conoce, que pueda asimilarla o insertarla en los aprendizajes que ya ha construido en el transcurso de sus experiencias previas de aprendizaje, esto quiere decir que sea potencialmente significativo desde el punto de vista psicológico. Esta "significatividad psicológica" se refiere al conocimiento previo del estudiante como factor decisivo en el momento de afrontar la adquisición de nuevos conocimientos.

Además de lo anterior, se requiere que el estudiante tenga una actitud favorable para aprender significativamente, esto hace referencia a una intencionalidad del estudiante para relacionar el nuevo material de aprendizaje con lo que ya conoce, con los conocimientos adquiridos previamente. Cuando la intencionalidad es escasa, éste se delimitará probablemente a memorizar lo aprendido de una forma mecánica y repetitiva; por el contrario, cuando la intencionalidad es elevada, el estudiante establecerá múltiples y variadas relaciones entre lo nuevo y lo que ya conoce.

Lo anterior depende de la motivación "que éste tiene para aprender significativamente y de la habilidad del docente para despertar e incrementar esa motivación" (Diaz Barriga, 1998, p.74). La intervención del profesor en este sentido es un factor determinante, pues la memorización mecánica y repetitiva de lo aprendido suele aparecer en un principio como un procedimiento mucho más cómodo y económico en tiempo y energía para el estudiante que la construcción de significados mediante la búsqueda y el establecimiento de relaciones sustantivas entre lo nuevo y lo que ya conoce.

El aprendizaje significativo implica, segun Ausubel (19976) su "memorización compresiva, su ubicación o almacenamiento en una red más o menos amplia de significados. Así mismo, en la medida en que contribuye a ampliar y extender dicha red de significados, se incrementa la capacidad del estudiante para establecer nuevas relaciones cuando se enfrenta a posteriores tareas o situaciones, por lo que un aprendizaje realizado de forma

\section{1}


significativa, es al mismo tiempo, un aprendizaje que tiene un elevado valor funcional, es decir, un aprendizaje útil, que puede ser utilizado para generar nuevos significados" (p.310).

Para Ausubel, el concepto de aprendizaje significativo implica un cambio de perspectiva en la solución dada al clásico problema pedagógico de la preparación o disponibilidad para el aprendizaje. El énfasis ya no reside en la potencia intelectual de estudiantes, directa o indirecta, relacionada con su nivel de desarrollo evolutivo, sino más bien en la existencia de conocimientos previos pertinentes para el contenido de aprender, que depende, por supuesto, en parte de dicha competencia intelectual, pero también, y sobre todo, de las experiencias previas del aprendizaje, tanto en la institución como fuera de ella. Pero el concepto de aprendizaje significativo supone, ante todo, un cambio de perspectiva radical en la manera de entender el proceso enseñanza aprendizaje. Frente a la concepción habitual y tradicional de que el aprendizaje del estudiante depende directamente de la influencia del profesor y de la metodología de enseñanza utilizada, se pone de relieve la importancia del conocimiento previo del estudiante y en general de sus procesos de pensamiento.

Todo parece indicar que el estudiante construye significados al mismo tiempo que atribuye un sentido a lo que aprende, de tal manera que las significaciones que finalmente construye a partir de lo que se le enseña no depende sólo de los conocimientos previos que posea y de su puesta en relación con el nuevo material de aprendizaje, sino también del sentido que atribuye a éste y a la propia actividad de aprendizaje.

Para los docentes, las estrategias para activar o generar conocimientos previos y para establecer expectativas adecuadas en los estudiantes, son aquellas estrategias dirigidas a activar los conocimientos previos o incluso a generarlos cuando no existan. En este grupo se pueden incluir también aquellas otras que se concentran en el esclarecimiento de las intenciones educativas que el profesor pretende lograr al término del ciclo o situación educativa.

La activación del conocimiento previo puede servir al profesor en un doble sentido: para reconocer lo que saben sus alumnos y para utilizar tal conocimiento como base para promover nuevos aprendizajes.

El establecer a los alumnos las intenciones educativas $u$ objetivas y objetivos, les ayuda a desarrollar expectativas adecuadas sobre el curso, y a encontrar sentido $\mathrm{y} / \mathrm{o}$ valor funcional a los aprendizajes involucrados en el curso.

En cuanto a las estrategias para orientar la atención de los alumnos, "son aquellos recursos que el profesor o el diseñador utiliza para focalizar y mantener la atención de los aprendices durante una sesión, discurso o texto. Los procesos de atención selectiva son actividades fundamentales para el desarrollo de cualquier acto de aprendizaje de tipo construccional, dado que pueden aplicarse de manera continua para indicar a los alumnos sobre qué puntos, conceptos o ideas deben centrarse los procesos de atención, codificación y aprendizaje.

Algunas estrategias que pueden incluirse en este son las siguientes: las preguntas insertadas, el uso de pistas o claves para explotar distintos índices estructurales del discurso - ya sea oral o escrito - y el uso de ilustraciones" (Ausubel, 19976, p.407).

Las estrategias para organizar la información que se ha de aprender permiten dar mayor contexto organizativo a la información nueva que se aprenderá al representarla en forma gráfica o escrita. Proporcionar una adecuada organización a la información que se ha de aprender, como ya hemos visto, mejora su significatividad lógica, y en consecuencia, hace más probable el aprendizaje significativo de los alumnos.

Y por último, las estrategias para promover el enlace entre los conocimientos previos y la nueva información que se ha de aprender, "son aquellas destinadas a crear o potenciar enlaces adecuados entre los conocimientos previos y la información nueva a aprenderse, asegurando con ello una mayor significatividad de los aprendizajes logrados: a este proceso de integración entre los "previos y los "nuevos" se le denomina "construcción de conexiones externas".

\section{CONCLUSIONES.}

El aprendizaje significativo es de gran importancia en el proceso educativo y es el mecanismo humano por excelencia para adquirir y almacenar la vasta cantidad de ideas e información representadas por cualquier campo del conocimiento.

Desde esta perspectiva, el docente universitario debe buscar las estrategias pertinentes para lograr un nivel apropiado de comprensión por parte de los educandos; debe cambiar de actitud y comprender que no puede construir conocimiento copiando las metodologías con las que él fue formado; y debe admitir que la visión constructivista proporciona diversas alternativas de enfocar y orientar los procesos de aprendizaje en una actividad planeada, intencionada y dinámica que propicie el desarrollo cognitivo y por consiguiente la construcción de conocimiento.

\section{REFERENCIAS BIBLIOGRÁFICAS}

AUSUBEL, David (1976): Sicología Educativa, un punto de vista cognoscitivo. $10^{\circ}$ edición. México: Editorial Trillas.

BORJA, Andrés (1990): La construcción de aprendizajes significativos en ciencias sociales. Barranquilla: Universidad Libre.

DIAZ BARRIGA, Frida (1998): Piaget en la educación: Debate en torno a sus aportaciones. $1^{\circ}$ edición. México D.F: Editorial Paidós. 